\title{
Characteristics of Saturn's FUV aurora observed with the Space Telescope Imaging Spectrograph
}

\author{
Jean-Claude Gérard, Denis Grodent, Jacques Gustin, and Adem Saglam \\ Laboratoire de Physique Atmosphérique et Planétaire, Université de Liège, Liège, Belgium
}

John T. Clarke

Center for Space Physics, Boston University, Boston, Massachusetts, USA

John T. Trauger

Jet Propulsion Laboratory, Pasadena, California, USA

Received 30 March 2004; revised 27 May 2004; accepted 28 June 2004; published 22 September 2004.

[1] We analyze a set of 15 FUV images obtained between October 1997 and January 2001 with the Hubble Space Telescope Imaging Spectrograph (STIS), providing a good view of Saturn's south auroral oval. It is found that the morphology and brightness distribution of the aurora are dynamical with variations occurring on time scales of hours or less. The dayside main oval lies between $70^{\circ}$ and $80^{\circ}$ and is generally brighter and thinner in the morning than in the afternoon sector. The afternoon sector is characterized by more diffuse emission at higher latitudes. Weak emission is also observed poleward of the main oval up to the pole. A spot of enhanced auroral precipitation, tentatively identified as the optical signature of the dayside cusp, is sometimes observed poleward of the main oval in the noon sector, especially during periods when the morning arc is not fully developed. A spiral structure of the main oval with arcs at two latitudes in the same sector is occasionally observed. The brightness of the main oval ranges from below the STIS threshold of $1 \mathrm{kR}$ of $\mathrm{H}_{2}$ emission up to $\sim 75 \mathrm{kR}$. The total electron precipitated power varies between 20 and $140 \mathrm{GW}$, that is, comparable to the Earth's active aurora but about two orders of magnitude less than on Jupiter. An increasing trend of the precipitated power between the 1997 and the 2000-2001 observations may be related to the rising solar activity. Six spectra of the aurora in the noon sector covering the 1200-1700 A range are dominated by emissions of the Lyman- $\alpha$ line and $\mathrm{H}_{2}$ Werner and Lyman bands. Their comparison with a synthetic model of electron excited $\mathrm{H}_{2}$ emissions indicates the presence of a weak absorption below $1400 \AA$ by a column of methane ranging between $7 \times 10^{15}$ and $2 \times 10^{16} \mathrm{~cm}^{-2}$. The corresponding energy of the primary auroral electrons is estimated $12 \pm 3 \mathrm{keV}$, using a low-latitude model atmosphere based on Voyager occultation measurements. The main oval brightness and the characteristic electron energy are generally consistent with recent models of Saturn's aurora, which colocate the main oval with the narrow upward field-aligned current system associated with departure from plasma corotation near the open-closed field line boundary. The latitude of the bright morning arc is somewhat lower than model predictions based on the plasma flow velocity measured by Voyager in the middle magnetosphere. INDEX TERMS: 2756 Magnetospheric Physics: Planetary magnetospheres (5443, 5737, 6030); 2704 Magnetospheric Physics: Auroral phenomena (2407); 2716 Magnetospheric Physics: Energetic particles, precipitating; 2724 Magnetospheric Physics: Magnetopause, cusp, and boundary layers; 6275 Planetology: Solar System Objects: Saturn; KEYWORDS: ultraviolet aurora, Saturn, magnetosphere, electron precipitation, spectroscopy, electron energy

Citation: Gérard, J.-C., D. Grodent, J. Gustin, A. Saglam, J. T. Clarke, and J. T. Trauger (2004), Characteristics of Saturn's FUV aurora observed with the Space Telescope Imaging Spectrograph, J. Geophys. Res., 109, A09207, doi:10.1029/2004JA010513.

\section{Introduction}

[2] Saturn's aurora and magnetosphere are intermediate between the solar wind driven case of the Earth and the

Copyright 2004 by the American Geophysical Union. 0148-0227/04/2004JA010513\$09.00 dominance of corotating plasma at Jupiter and thereby key to understanding the effects of the solar wind on giant planet magnetospheres. Unlike Jupiter or the Earth, the magnetic dipole of Saturn is closely aligned with the planetary spin axis with an offset angle less than $1^{\circ}$ [Connerney et al., 1984; Davis and Smith, 1990]. Like its terrestrial counter- 
part that constantly adjusts to the magnitude and orientation of the interplanetary magnetic field (IMF), Saturn's aurora shows large intensity and latitudinal variations of the main oval, suggesting a solar wind controlled aurora. The source of plasma in its magnetosphere is thought to be mostly external (solar wind), although internal sources such as the rings, icy satellites, and Titan may also contribute. Saturn's aurora has received much less attention than its Jovian counterpart because of the larger distance from the Earth, smaller angular extent, and intrinsically fainter emission.

[3] Until recently, most of our knowledge concerning the morphology of the far ultraviolet (FUV) aurora was based on data collected with the Voyager UV spectrometer (UVS) during the Voyager 1 and 2 flybys of Saturn in 1980. The Saturnian aurora appeared in the Voyager data as a narrow circumpolar region with no apparent emission present in the polar cap [Broadfoot et al., 1981; Sandel and Broadfoot, 1981], probably originating from the distant magnetosphere. At Saturn, as at Jupiter, both HI Lyman- $\alpha$ and $\mathrm{H}_{2}$ Lyman and Werner bands are present in the polar regions of both hemispheres. A typical intensity of 10 to $15 \mathrm{kR}$ was estimated for the total $\mathrm{B}-\mathrm{X}$ and $\mathrm{C}-\mathrm{X} \mathrm{H}_{2}$ emission [Broadfoot et al., 1981] but, like its terrestrial counterpart, Saturn's aurora exhibits large intensity temporal variations (factors of $\sim 2-5$ ) [Sandel et al., 1982], as is expected for a solar wind controlled aurora. A systematic dependence on Saturn's longitude was also suggested with peaks near $\lambda_{\mathrm{SLS}} \sim 135^{\circ}$ and brightening occurring when the Sun was near $\lambda_{\mathrm{SLS}} \sim 100^{\circ}$ [Sandel et al., 1982]. It has been argued that these brightness variations are related to the Saturn kilometric radiation (SKR), whose source also lies near $80^{\circ}$ latitude at longitudes between $0^{\circ}$ and $120^{\circ}$. Different auroral emissions have been observed to exhibit both local time and longitudinally fixed variations. Radio wave observations during the Voyager encounters [Kaiser et al., 1984] indicated that variations of the SKR emission in the auroral zones are strongly correlated with solar wind pressure changes. The UV auroral emissions observed with Voyager UVS also appeared correlated with both the SKR intensity and the longitude of Saturn facing the solar wind, suggesting an active sector in the magnetosphere. No further knowledge of the morphology of Saturn's aurora was gathered from observations made with the International Ultraviolet Explorer (IUE). The $\mathrm{H}_{2}$ spectrum was below the detection limit, but outbursts of Lyman- $\alpha$ were intermittently observed over a decade with a brightness of a few kiloRayleighs [Clarke et al., 1981; McGrath and Clarke, 1992]. The Faint Object Camera on board HST was used to obtain the first image of the north Saturnian aurora, with all images coadded to increase the signal to noise ratio [Gérard et al., 1995]. The speculated relationship between the aurora and the polar haze was confirmed by the analysis of the UV polar darkening observed with pre-COSTAR [Ben Jaffel et al., 1995] and post-COSTAR UV [Gérard et al., 1995] Faint Object Camera images of the north polar region.

[4] A set of Wide Field Planetary Camera (WFPC2) FUV images [Trauger et al., 1998] with a higher limiting sensitivity $(\sim 5 \mathrm{kR})$ showed a northern auroral arc that appeared fixed in local time near the dawn limb. This is consistent with the dawn auroral storms observed on Jupiter and appears to be a common feature of the giant planet magnetospheres. The brightness of the emission was quite variable
$(<5 \mathrm{kR}$ to $90 \mathrm{kR})$, but the morning sector was consistently enhanced in comparison with the afternoon sector. Modeling of the auroral curtain indicated that the latitude of the auroral oval was between $74^{\circ}$ and $79^{\circ} \mathrm{N}$, consistent with the earlier Voyager results and supporting a mapping into the distant magnetosphere. Infrared emissions from the ionospheric $\mathrm{H}_{3}^{+}$ion have also been detected from the ground [Geballe et al., 1993] and have been shown to be mainly auroral in nature [Stallard et al., 1999]. Recently, Cowley et al. [2004a] described an image taken on 8 December 2000 with the Space Telescope Imaging Spectrograph (STIS). It shows the presence of a discrete auroral oval extending continuously from the midnight sector via dawn into the postnoon hours, though strongly varying in brightness with local. The latitude of the emission also clearly changed with local time, lying at $\sim 72^{\circ}$ at dawn, increasing to $\sim 75^{\circ}$ at noon, and to $\sim 78^{\circ}$ in the early afternoon.

[5] An estimate of the altitude of the aurora relative to the hydrocarbon homopause may be derived from the FUV color ratio. This color ratio $\mathrm{CR}=\mathrm{I}(1557-1619 \AA) / \mathrm{I}(1230$ $1300 \AA$ ) is related to the rapid drop in the $\mathrm{CH}_{4}$ absorption cross sections at wavelengths above $1400 \AA$, which leaves the bulk of the longer wavelength $\mathrm{H}_{2}$ Lyman band emissions unattenuated, while the $\mathrm{H}_{2}$ emissions at wavelengths $<1400 \AA$ are absorbed. Therefore the altitude of the auroral emitting layer relative to the methane homopause and the particle penetration depth (and thus the particle energy) can be derived from the observed ratio $\mathrm{CR}$ with suitable model atmosphere, particle energy deposition, and electron transport models. Low spectral resolution Voyager UVS spectra provided some indication on the nature and energy of the auroral energetic particles interacting with Saturn's atmosphere [Sandel et al., 1981; Sandel and Broadfoot, 1981; Shemansky and Ajello, 1983]. Self-absorption in some $\mathrm{H}_{2}$ bands was observed in a bright spectrum ( $100 \mathrm{kR})$, suggesting that the auroral source was located deeper in the atmosphere than the low-latitude emission $\left(\mathrm{H}_{2}\right.$ density on the order of $2 \times 10^{11} \mathrm{~cm}^{-3}$ ). Weak spectra appeared to originate near the exobase and required no $\mathrm{CH}_{4}$ absorption, but the brighter spectrum was best fitted with an overlying methane column density of $8 \times 10^{15} \mathrm{~cm}^{-2}$. The spectrum was compatible with an emission induced by energetic electron impact. However, the possibility has been raised of $\mathrm{N}$ ion precipitation following the escape of nitrogen atoms from Titan into Saturn's magnetosphere. The STIS instrument, with its unique spectral imaging facility and increased sensitivity, offers the possibility to obtain spatially resolved information on the ultraviolet color ratio and directly compare them with the FUV auroral emission morphology.

[6] In this study we analyze the morphology of Saturn's FUV aurora based on observations made with STIS. The observations were obtained during a period of good viewing geometry of the planet's south pole, although in 1997 emission was also observed close to the planet's northern polar limb. We describe the auroral distribution in the south and show polar projections. The variability is important both in morphology and brightness of the aurora. We also show that the total electron precipitated power varies by a factor of $\sim 5$, probably as a result of changes in the solar wind characteristics. Finally, we show that the weak hydrocarbon absorption in FUV spectra is compatible with 
Table 1. Characteristics of STIS Images of Saturn's FUV Aurora ${ }^{a}$

\begin{tabular}{|c|c|c|c|c|c|c|c|}
\hline Image - Archival Nr & $\mathrm{Nr}$ & UT & CML, deg & Filter & Exposure, $\mathrm{s}$ & Sub-Earth Latitude, deg & Emitted Power, GW \\
\hline \multicolumn{8}{|c|}{11 October 1997} \\
\hline o4bd01ycq & 1 & 0357 & 98.4 & SRF2 & 564 & -10.3 & 5.4 \\
\hline o4bd01yeq & 2 & 0422 & 112.1 & _ & 774 & -10.3 & 7.5 \\
\hline o4bd02zrq & 3 & 0715 & 209.4 & SRF2 & 1000 & -10.3 & 1.8 \\
\hline o4bd02ztq & 4 & 0736 & 221.0 & - & 755 & -10.3 & 3.0 \\
\hline o4bd03awq & 5 & 1029 & 318.6 & SRF2 & 1000 & -10.3 & 1.5 \\
\hline o4bd03ayq & 6 & 1049 & 330.0 & - & 754 & -10.3 & 4.4 \\
\hline \multicolumn{8}{|c|}{20 November 1997} \\
\hline o4bd04vmq & 7 & 1334 & 91.9 & SRF2 & 1000 & -8.9 & 7.5 \\
\hline o4bd04voq & 8 & 1356 & 104.6 & - & 1000 & -8.9 & 10.5 \\
\hline \multicolumn{8}{|c|}{5 December 1997} \\
\hline o4bda $5 \mathrm{kcq}$ & 9 & 0536 & 103.2 & - & 600 & -8.7 & 4.8 \\
\hline \multicolumn{8}{|c|}{7 December 2000} \\
\hline o5dta2nyq & 10 & 1134 & 241 & SRF2 & 480 & -23.3 & 25.8 \\
\hline \multicolumn{8}{|c|}{8 December 2000} \\
\hline o5dta1tnq & 11 & 1004 & 281.1 & SRF2 & 480 & -23.3 & 6.1 \\
\hline \multicolumn{8}{|c|}{28 January 2001} \\
\hline o5hrb1v4q & 12 & 0850 & 184.7 & SRF2 & 330 & -23.1 & 7.2 \\
\hline o5hrb1viq & 13 & 1149 & 285.5 & SRF2 & 600 & -23.1 & 12.2 \\
\hline \multicolumn{8}{|c|}{29 January 2001} \\
\hline o $5 \mathrm{hra} 2 \mathrm{clq}$ & 14 & 1032 & 333.1 & SRF2 & 300 & -23.1 & 10.0 \\
\hline o5hra2cfq & 15 & 1332 & 74.2 & SRF2 & 600 & -23.1 & 14.0 \\
\hline
\end{tabular}

${ }^{\mathrm{a}}$ Times and central meridian longitudes are given at midexposure.

precipitation of $\sim 10 \mathrm{keV}$ auroral electrons if low-latitude atmospheric models are applicable to auroral regions. These results are compared with predictions based on recent models of electron acceleration in upward field-aligned currents.

\section{Observations}

[7] A total of 15 STIS images were obtained with the photon-counting detector 25MAMA (Multi-Anode Microchannel Array) during the period between 11 October 1997 and 28 January 2001. The STIS instrument was operated in the TIMETAG mode and provided an event stream with fast time resolution. However, all measured counts were integrated over the time of the exposure to improve the signal to noise ratio. The moving target mode was used to acquire the spectra, implying that the aperture was placed at a fixed location with respect to the planet and did not track a preset planetary longitude. The images were taken either in the MAMA clear mode or with the $\mathrm{SrF}_{2}$ filter rejecting Lyman- $\alpha$. In the clear mode the solar-blind detector has a bandpass ranging from 115 to $180 \mathrm{~nm}$ and is sensitive to the $\mathrm{H}_{2}$ Lyman and Werner bands as well as the strong $\mathrm{H}$ Lyman- $\alpha$ line. There are $1024 \times 1024$ pixels in the MAMA array and their size is 0.0243 arcsec, thus providing a field of view of $24.7 \times 24.7 \operatorname{arcsec}^{2}$ with a 0.08 arcsec full width at half maximum point spread function (PSF). The distance subtended by 1 pixel on the field of view projected at Saturn is then approximately $150 \mathrm{~km}$. At this distance a resolution element corresponds to $\sim 500 \mathrm{~km}$ on the planet. The planet's rotation introduces some smearing of the images for features in corotation. For a feature located at the central meridian longitude (CML) and at $70^{\circ}$ it is $0.56^{\circ}$ in $1 \mathrm{~min}$. The blurring is relatively less near the limb, where the rotational motion is more along the line of sight. The guiding of the STIS aperture was executed in fine lock mode using onboard acquisition.

[8] The $24.7 \times 24.7 \operatorname{arcsec}^{2}$ field of view of STIS captures the full planet's disk and a fraction of the ring system. A limb-fitting procedure is used to determine the position of the center of the planet. The procedure consis calculated for the appropriate viewing geometry. The coordinates are found by a chi-ts in fitting the sunlit planetary limb, the inner limit of the $\mathrm{C}$ ring, and the outer limit of the A ring with a model contour squares iterative process that minimizes the distance between the calculated and the observed contours. Simulations with synthetic planetary disks and noisy signal have shown that the final accuracy of this determination is approximately 1 STIS resolution element (3 pixels). Each pixel of the initial image is then assigned a planetocentric latitude and a planetary longitude. This procedure makes it possible to generate polar (orthographic) projections as if the observer was located above the south pole. It is possible to build maps in a system in corotation with the planet or fixed with respect to the central meridian. The STIS images span a period of over 3 years (Table 1) during which Saturn's sub-Earth latitude varied from $10.0^{\circ} \mathrm{S}$ to $23.1^{\circ} \mathrm{S}$, offering an increasingly complete view of the south auroral oval. The planetary longitude given in Table 1 refers to the S3 system. The uncertainty on the planetary rotation period is such that it is no longer possible to relate the longitudes observed at the times of the Voyager encounters with those currently used. A more accurate system is expected to be derived by the Cassini mission, with a conversion tied to the SLS system.

[9] The methodology used to process the images was described in detail by Grodent et al. [2003a] and will only be summarized here. As part of our data reduction process, 
the images were flat-fielded using the most recent loworder flat field and pixel-to-pixel files provided by the Space Telescope Science Institute (STScI). Dark counts were subtracted using the appropriate STScI dark files. The STIS/FUV-MAMA clear counts were converted in flux using a factor of $5.06 \times 10^{-3}$ count pix $^{-1} \mathrm{~s}^{-1}$ for $1 \mathrm{kR}$ in the $\mathrm{SrF}_{2}$ mode and $1.50 \times 10^{-4}$ count $\mathrm{pix}^{-1} \mathrm{~s}^{-1} / \mathrm{kR}$ in the clear mode. These conversion factors were calculated based on a synthetic UV spectrum of $\mathrm{H}_{2}$ [Gérard et $a l ., 2002]$, including the Lyman- $\alpha$ line contributing $\sim 15 \%$ of the total $\mathrm{H}_{2}$ intensity. Some uncertainty in identifying locations on the planet arises from the error in our determination of the planet center. The vertical extent of the auroral curtain has been neglected. The latitudes and longitudes were calculated assuming an oblate spheroid with a radius corresponding to an average height of the auroral emission of $1000 \mathrm{~km}$ above the 1 bar level. The uncertainty on latitudes associated with the positioning of the coordinate grid and the auroral vertical extent is estimated as $1.5^{\circ}$ in the 1200 MLT sector and less than $1^{\circ}$ in the dawn and dusk sectors. Similarly, the longitudinal inaccuracy is less than $1^{\circ}$ at noon and $5^{\circ}$ at 0600 and 1800 LT. These values are comparable with the level of pixel stretching in the polar projections presented below.

[10] Several far ultraviolet auroral spectra were also collected with STIS from July 1997 to January 2001. The spatially resolved spectra were imaged on the FUV-MAMA photon counting detector providing a $1024 \times 1024$ pixels matrix. The G140L grating, combined with the $25 \times$ $0.5 \operatorname{arcsec}^{2}$ long-slit, provided spectra in the $1100-1750$ $\AA$ range with $\sim 12 \AA$ resolution. Other MAMA spectra collected with the $52 \times 2 \operatorname{arcsec}^{2}$ aperture were not usable for this study. Some of the G140L spectra were preceded and/or followed by auroral FUV images as will be described in section 3.3.

\section{Results}

[11] We first describe the STIS images and the morphology and time variations determined from the comparison of the 15 images. We then analyze STIS spectra and examine the constraints on the auroral electrons that can be derived from the spectral characteristics.

\subsection{FUV Auroral Morphology}

[12] The 15 STIS images all show solar light reflected by the full planetary disk, its dark polar region, the rings and the polar aurora. The background counts outside the disk and rings are considerably lower in the exposures made with the $\mathrm{SrF}_{2}$ filter, since the geocoronal Lyman- $\alpha$ contribution is filtered out. All 15 images show the presence of auroral emissions in the south at least in some longitude sectors. Cowley et al. [2004a] described the image obtained on 7 December 2000. They found that the latitude of the emission increased from dawn to the 0700-0900 LT brightest region where the incident energy flux reached between 4 and $8 \mathrm{~mW} / \mathrm{m}^{2}$. The nine exposures made in 1997 also offer a partial view of the north aurora and all show some emission near the polar limb. The first six images were obtained on 11 December 1997 over a 412-min time span, covering $65 \%$ of Saturn's rotation. They illustrate the large intensity variations and the

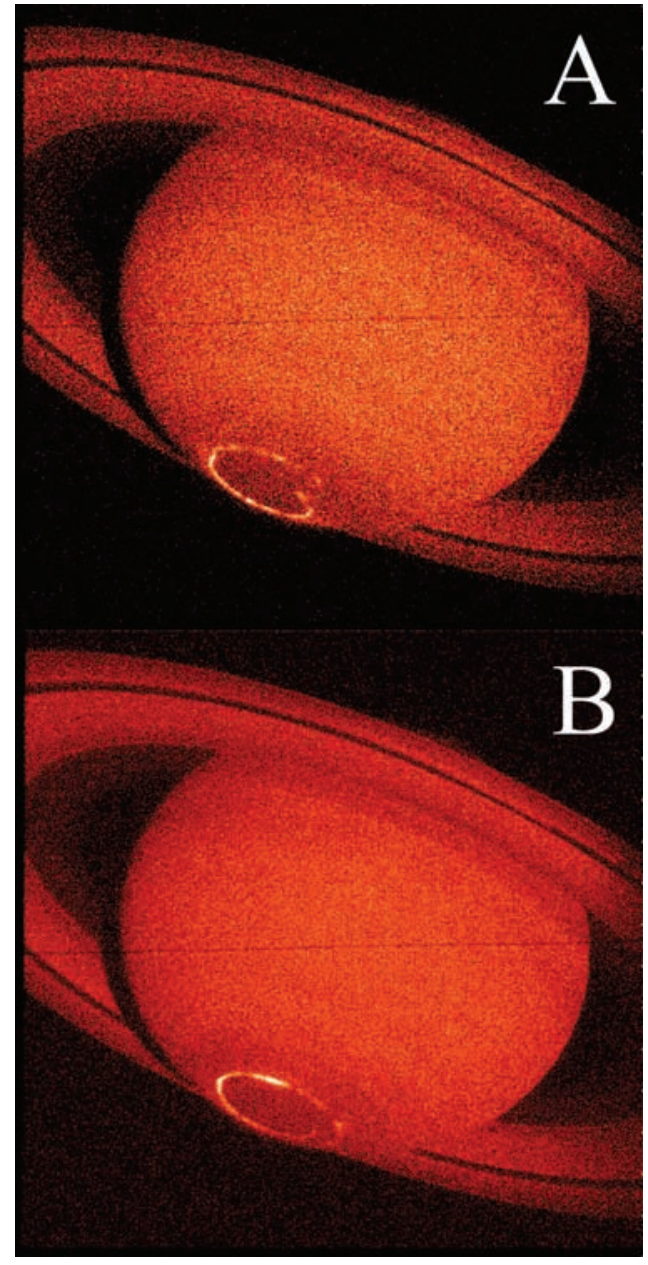

Figure 1. Examples of raw STIS images of Saturn taken on 29 January 2000 at 1032 and 1332 UT. Differences are observed in the global auroral morphology and brightness distribution over the 3 hours separating the two images.

dynamical nature of Saturn's FUV aurora. The two filtered images of 29 January 2001 appearing in Figure 1a were taken 3 hours apart and display an example of the dynamics of Saturn's changing morphology. The first image of this set shows a continuous thin oval extending from the midnight meridian to the dawn sector with maximum brightness along the nightside limb. In the afternoon sector a discontinuity is observed between the external oval continuing the dayside structure and an internal oval at significantly lower latitude. The main oval region therefore globally presents a spiral aspect. The same general morphology is seen in Figure 1b, with its spiral-like aspect and latitude discontinuity at about the same location as in Figure 1a. In this case, however, the external oval extends beyond the longitude of the end of the inside oval so that two regions of precipitation coexist at the same longitude in the dusk sector. Also, in the second image, a brightness enhancement is observed in the prenoon sector, separated from the morning ansa by a region of weaker auroral precipitation. The maximum emission rate in Figure 1a is about $50 \mathrm{kR}$, corresponding to a precipitated flux of about $5 \mathrm{~mW} / \mathrm{m}^{2}$ in the midnight 


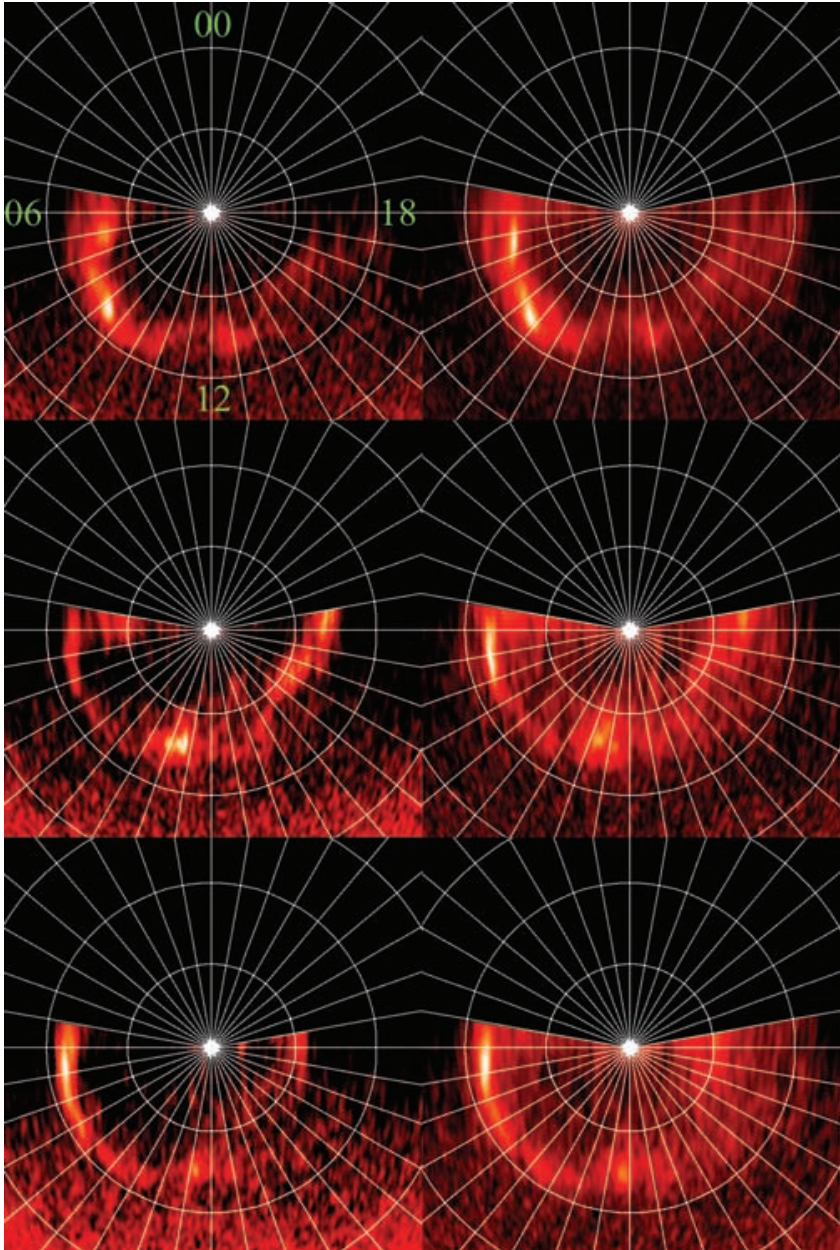

Figure 2. Sequence of six consecutive (from left to right and top to bottom) polar projections of images obtained on 11 October 1997 covering $65 \%$ of a planetary rotation. The pole is at the center and circles indicate the $80^{\circ}, 70^{\circ}$, and $60^{\circ}$ parallels. Noon (solar time) is at the bottom, midnight is at the top, dawn is at the left, and dusk is at the right. The meridians are spaced by $10^{\circ}$.

sector and $74 \mathrm{kR}\left(7.5 \mathrm{~mW} / \mathrm{m}^{2}\right)$ in the prenoon sector of Figure $1 \mathrm{~b}$.

[13] Polar orthographic projections of the 15 images were made to analyze the morphology of the dayside aurora. Figure 2 shows a set of six polar projections for the 11 October 1997 observations. Nightside emission, although partly visible on raw images, are too close to the limb, causing a considerable spreading in latitude and uncertainty on the location once projected. We therefore generally restrict our analysis to a sector extending $100^{\circ}$ on either side of the noon meridian. At 0357 UT a weak continuous oval is observed with a brightening near the dawn limb. At 0715 UT the morning oval enhancement has vanished to reappear at 1029 UT, closer to the central meridian. In the intermediate images, a bright spot is observed near local noon and a complete nearly homogeneous oval is observed. Finally, at 1049 UT the noon sector is enhanced and some weak emission is observed at higher latitude, inside the oval. These observations confirm that significant variations of the brightness distribution are observed on time scales as short as
20 min. Five of the six images show a bright ( 8 to $70 \mathrm{kR}$ ) region of emission extending from dawn to approximately $40^{\circ}$ (160 min of local time) from local noon, although its extension toward noon is more limited in the second, third, and fourth images. The latitude of the maximum emission changes from $77^{\circ}$ at 1800 LT on the first image to $73^{\circ}$ on the fifth image. The latitude of this morning arc slightly decreases as local time increases, suggesting either that the arc does not form along a constant L-shell or that the magnetic field near the planet departs from the axis symmetry indicated by models of Saturn's magnetic field.

[14] An interesting feature is observed on all six images near the noon sector where a bright spot, somewhat disconnected from the main oval, is observed. Its center (brightest pixels) is located poleward of the main oval, near $75^{\circ} \mathrm{S}$ at longitudes between $16^{\circ}$ westward and $10^{\circ}$ eastward of the noon meridian. Its brightness (10 to $20 \mathrm{kR}$ ) may be nearly as high as the main oval. The presence of a bright spot in the noon sector is observed in 9 of the 15 auroral images. It is present on 4 out of 7 days, that is, an occurrence frequency of $\sim 60^{\circ}$. Remarkably, this feature is not present (or below the sensitivity threshold of $\sim 1 \mathrm{kR}$ ) when the dayside oval is reduced to a thin arc stretching along $70^{\circ} \mathrm{S}$ from the dawn to the noon meridians, such as in images $10,11,12,14$, and 15. It thus appears that Saturn's auroral morphology varies between the two extreme cases illustrated in Figure 3. In Figure $3 \mathrm{a}$ the oval has been projected at all local times since its nightside segment is close to the pole and Saturn's bright ring does not significantly interfere with the auroral emission. The projections of the STIS nightside pixels are elongated by as much as $\sim 5^{\circ}$ along the noon-midnight meridian, as they are located close to the planetary limb. The projection shows a pattern characterized by an arc whose latitude decreases from $83^{\circ}$ in the midnight sector, to $74^{\circ}$ at dawn, and $70^{\circ}$ at noon, accompanied by weaker higher-latitude emissions in the afternoon and dusk sectors, such as in images 13,14 , and 15 . In addition, a spiral shape is clearly seen, and we note that in the dusk sector two arcs are simultaneously observed with a longitudinal overlap of $\sim 30^{\circ}$ of longitude ( 2 hours of local time). In Figure $3 \mathrm{~b}$ a weak emission is located in the morning sector with a bright noon spot showing a large latitudinal extent. Additional diffuse precipitation is observed poleward of $70^{\circ}$, up to the south pole. An area nearly void of emission is seen in a region bounded equatorward by the main oval and extending up to $80^{\circ}$ in the dawn sector and $70^{\circ}$ at 1000 LT. This region is reminiscent of the dark polar region identified in infrared [Stallard et al., 2003] and FUV [Grodent et al., 2003a] images of the Jovian aurora.

[15] The sum of 9 of the 10 exposures taken with the $\mathrm{SrF}_{2}$ filter is shown in Figure 4. Image 10 was not included as it shows an unusually bright region of emission in the prenoon sector, possibly resulting from temporarily disturbed solar wind conditions. This average Saturnian auroral morphology confirms the prominence of the morning arc. The equatorward boundary of the aurora is close to $70^{\circ}$. The main arc extends from dawn to near noon at $71^{\circ}-72^{\circ}$ with a pronounced brightening in the prenoon region and drops considerably as the arc approaches the noon sector. In the afternoon sector the emission is more diffuse, more widespread, and dimmer than in the morning region. It progressively vanishes to reach the background level at dusk. The 


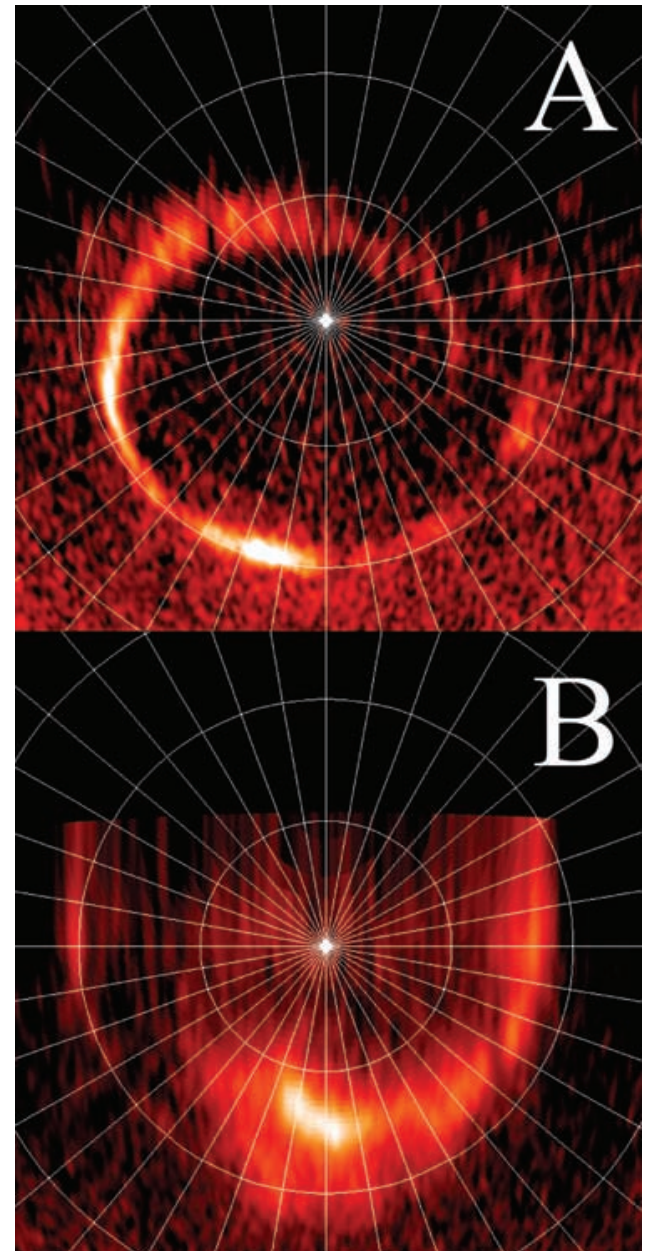

Figure 3. Two examples of morphology of Saturn's aurora. (top) A global spiral structure surrounds the north pole. The image pixels are stretched in the nightside sector where the auroral oval is close to the limb. A thin arc is observed in the morning sector with weak afternoon emission and no visible noon spot (29 January 2001, 1332 UT). (bottom) The morning arc is weak but a bright spot is observed near local noon extending from $73^{\circ}$ to $79^{\circ}$ and diffuse emission extends duskward near $74^{\circ}$ (20 November 1997, 1334 UT).

latitudinal width in this sector is typically $3-4^{\circ}$, in contrast with the thinner $\left(1^{\circ}\right.$ or less) morning arcs. Statistically, Figure 4 indicates that the auroral precipitation in the morning sector is $\sim 5$ times as large as in the afternoon. The bright spot in the noon sector is statistically located near $75^{\circ} \mathrm{S}$ but its contour is less clearly defined in the sum than in individual images. This is a consequence of its changing position in latitude and local time, as seen from individual images.

\subsection{Precipitated Power}

[16] The set of auroral images may be used to determine the electron precipitated energy flux and discuss its variations. This calculation is easier for images obtained with the $\mathrm{SrF}_{2}$ filter as both the geocoronal and the auroral Lyman- $\alpha$ emission is blocked out so that only auroral $\mathrm{H}_{2}$ and disk background contributions are detected. The total count rate above background may be converted in units of a total emitted power in the STIS passband. Using a measured spectrum of Saturn's aurora described later, the total $\mathrm{H}_{2}$ emission in the Lyman and Werner bands may be calculated. Model calculations [Gérard and Singh, 1982; Waite et al., 1983; Grodent et al., 2001] indicate that approximately $10 \mathrm{kR}$ are emitted in the two singlet states per incident $\mathrm{mW} / \mathrm{m}^{2}$, corresponding to $\mathrm{a} \sim 10 \%$ conversion efficiency between the electron precipitated power and the $\mathrm{H}_{2}$ band emission. Figure 5 shows the radiated power in the $\mathrm{H}_{2}$ Lyman and Werner bands associated with each image. The radiated power ranges from 2 to $14 \mathrm{GW}$, corresponding to a hemispheric precipitated power of 2 to $14 \times 10^{10} \mathrm{~W}$, with an exceptionally bright case at $26 \mathrm{GW}$ observed on 7 December 2000 . These values are typically two orders of magnitude less than the nearly constant $1 \times 10^{13} \mathrm{GW}$ measured in the Jovian aurora [Grodent et al., 2003b]. Comparing the values measured in 2001 with those observed in 1997, it may be argued that the mean value increased by a factor of $\sim 2$ over the 38 -month time period. This enhancement is possibly linked to the change of solar activity between a relatively moderate level in October 1997 (F10.7 index = 85) and the more active sun of January 2001 (F10.7 = 167). However, it is also possible that the larger planetary tilt angle (Table 1), offering a better visibility of the nightside oval, accounts for all or part of this apparent increase in radiated power.

\subsection{Energy of Auroral Electrons}

[17] STIS spectra obtained in the G140L mode with the $52 \times 0.5 \operatorname{arsec}^{2}$ aperture have been analyzed and fitted with synthetic spectra to calculate their FUV color ratio and determine whether they show a signature of absorption by

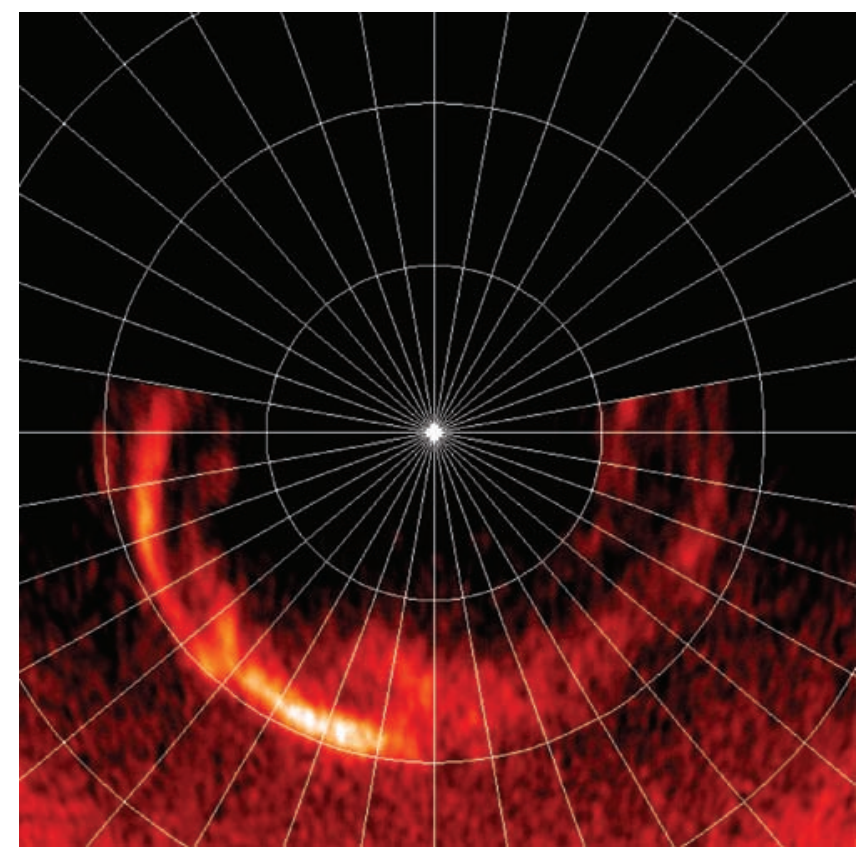

Figure 4. Average morphology of Saturn's south aurora obtained by summing nine polar projections of images obtained with the $\mathrm{SrF}_{2}$ filter. The pole is at the center and circles indicate the $80^{\circ}, 70^{\circ}$, and $60^{\circ}$ parallels. Noon (solar time) is at the bottom, midnight is at the top, dawn is at the left, and dusk is at the right. The meridians are spaced by $10^{\circ}$. 


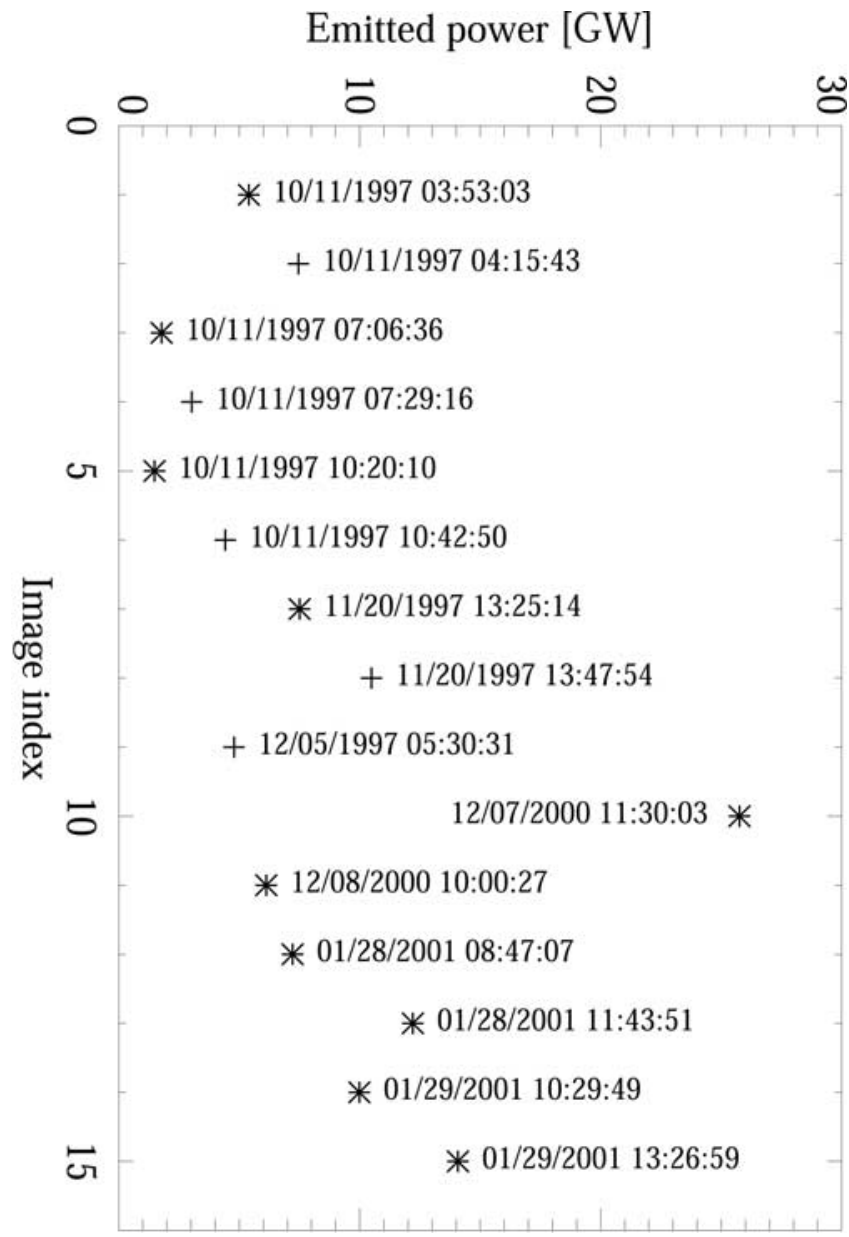

Figure 5. Auroral radiated power determined from the set of STIS images. The stars correspond to filtered $\left(\mathrm{SrF}_{2}\right)$ images, and the pluses correspond to unfiltered exposures. The error bars associated with the count statistics are less than their symbol size.

hydrocarbons. A total of six spectra were observed on 7 and 8 December 2000 and are available from the STScI archive database (Table 2). The background subtraction method and processing of the STIS spectra were described by Gustin et

Table 2. Characteristics of STIS Spectra of Saturn's FUV Aurora ${ }^{a}$

\begin{tabular}{|c|c|c|c|c|c|c|}
\hline $\begin{array}{c}\text { Spectrum } \\
\text { (Archive File Nr) }\end{array}$ & $\mathrm{Nr}$ & UT & $\begin{array}{c}\text { CML, } \\
\text { deg }\end{array}$ & $\begin{array}{c}\text { Exposure, } \\
\mathrm{s}\end{array}$ & $\begin{array}{l}\text { Color } \\
\text { Ratio } \\
\end{array}$ & $\begin{array}{c}\text { Main Oval } \\
\text { Average } \\
\text { Brightness, } \\
\text { kR } \\
\end{array}$ \\
\hline \multicolumn{7}{|c|}{7 December 2000} \\
\hline $05 \mathrm{dta} 2 \mathrm{o} 0 \mathrm{q}$ & 1 & 1153 & 253 & 1095 & 1.43 & 7.7 \\
\hline $05 \mathrm{dta} 2 \mathrm{o} 6 \mathrm{q}$ & 2 & 1325 & 305 & 1350 & 1.31 & 14.0 \\
\hline 05dta2ocq & 3 & 1501 & 0 & 1350 & 1.35 & 8.1 \\
\hline \multicolumn{7}{|c|}{8 December 2000} \\
\hline $05 \mathrm{dta} 1 \mathrm{tpq}$ & 4 & 1023 & 294 & 1095 & 1.39 & 6.3 \\
\hline 05dta1tpq & 5 & 1154 & 345 & 1350 & 1.39 & 4.8 \\
\hline 05dtalulq & 6 & 1330 & 39 & 1350 & 1.48 & 3.3 \\
\hline Mean & & & & & 1.39 & 7.4 \\
\hline
\end{tabular}

${ }^{a}$ Times and central meridian longitudes are given at midexposure.

${ }^{b}$ These values refer to the emission rate of the $\mathrm{H}_{2}$ Lyman and Werner bands at the intersection of the STIS slit and the dayside main oval latitudinally averaged over the width of the aurora. al. [2002, 2004] for Jovian spectra and will not be repeated here. The 1150-1750 $\AA$ spectral range includes both unabsorbed and absorbed $\mathrm{H}_{2}$ FUV emissions [see Gérard et al., 2003, Figure 1], which are used to derive color ratios. All spectral observations were made in the "time-tag" mode, without additional filters. The slit was positioned close to the central meridian, although the absolute pointing accuracy is not better than 1-2 arcsec. Filtered image 10 was obtained on 7 December 2000 only 19 min before spectrum 1. Similarly, image 11 on 8 December was collected 19 min before spectrum 4 . To locate the slit of a given spectrum on the related image, we use the method described by Gustin et al. [2002]. A number of columns of the image corresponding to the width of the slit are summed, and the resulting intensity curve is compared with the intensity obtained along the slit by integrating the spectrum longward of Lyman- $\alpha$. The location of the slit on the image is then moved until a good match is obtained. The accuracy of this method is estimated to be approximately 5 pixels $(0.1 \operatorname{arcsec})$, assuming that the auroral morphology does not significantly change between the two measurements. Figure 6 shows the result of the comparison between image 10 and spectrum 1, with the position and width of the slit marked by two parallel lines. It is found that the spectrum was taken close to the central meridian so that the spectrum is characteristic of local noon conditions. Similarly, it is found that spectrum 4 matches very closely the width of the oval along the central meridian. Since the nominal pointing was identical for the set of six spectra, it may be concluded that all were taken close to local noon.

[18] Figure 7 shows spectrum 1 taken on 7 December 2000. A synthetic $\mathrm{H}_{2}$ spectrum has been generated for a rovibrational temperature of $400 \mathrm{~K}$, close to the value measured in the Voyager occultation near the homopause [Smith et al., 1983]. It was convolved with the instrumental line spread function (LSF) following the method used for the analysis of STIS Jovian auroral spectra [Dols et al., 2000; Gérard et al., 2003; Gustin et al., 2002, 2004]. Simulations have shown that the value of the rovibrational

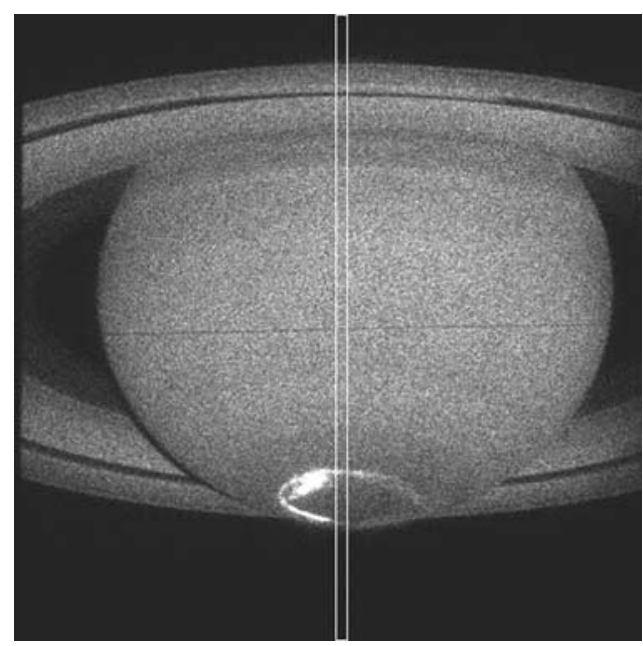

Figure 6. Image obtained at 1134 UT on 7 December 2000 (image 10 in Table 1) with the STIS MAMA aperture. The position of the STIS slit is marked by the double line crossing the polar region the planetary disk. 


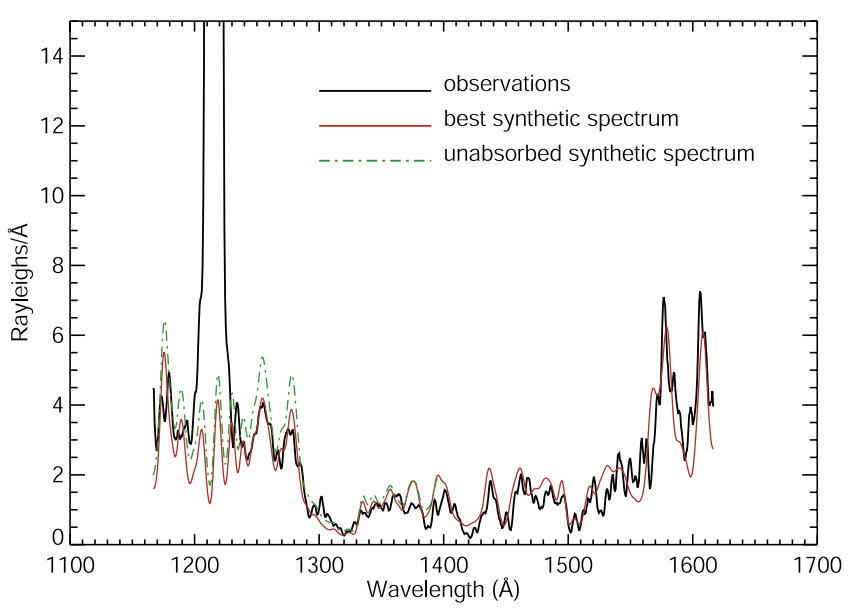

Figure 7. STIS spectrum obtained on 7 December 2000 at 1153 UT. The solid line shows the calibrated spectrum in Rayleighs/A smoothed on 5 pixels ( 2 A). The dotted line shows the best fit to a $\mathrm{H}_{2}$ synthetic spectrum for a methane slant column abundance of $9 \times 10^{15} \mathrm{~cm}^{-2}$. For comparison the dashed-dotted line shows the same spectrum calculated without $\mathrm{CH}_{4}$ absorption.

temperature does not play a significant role in the spectral distribution at the spectral resolution of this study [Dols et al., 2000, Figure 6].

[19] As mentioned before, the CR is a measure of the absorption of the $\mathrm{H}_{2}$ auroral emission by hydrocarbons located above or inside the region of the auroral energy deposition. It is thus related to the penetration of energetic electrons into the hydrocarbon layer and provides an indirect measure of the energy of the precipitated electrons in $\mathrm{H}_{2}$ atmosphere. The relationship between the FUV color ratio and the electron mean energy depends on the altitude distribution of hydrocarbons and require a model atmosphere. The relation between the mean electron energy $E$ of the auroral electrons and their penetration depth has been obtained for Jupiter, using a model Jovian atmosphere [Grodent et al., 2001]. For a given electron energy, the penetration depth will be identical on both planets where the dominant constituent is $\mathrm{H}_{2}$ in the two cases. One can thus associate a mean auroral electron energy with an observed color ratio, using a model of Saturn's atmosphere, if the methane vertical distribution in Saturn's atmosphere is known. The model by Moses et al. [2000] is used to relate the total pressure with temperature, altitude, and $\mathrm{CH}_{4}$ density. For the region near and above the homopause, it is based on the analysis of Voyager 2 stellar and solar occultation by Smith et al. [1983]. Simulations [Gérard et al., 1998] have shown that for a given precipitated auroral energy spectrum, the observed color ratio only moderately depends on the view angle. The view angle for the main oval is about $55^{\circ}$ at the central meridian, for the $7-$ 8 December 2001 period considered here.

[20] Table 2 lists the times, central meridian longitudes, and color ratios determined from each spectrum. The CR uncertainty, calculated from the total count rate in the numerator and denominator of the color ratio definition is estimated to be on the order of $\sim-0.1$. The $C R$ values range from 1.31 to 1.48 with a mean value of 1.39 , corresponding to a nearly constant vertical $\mathrm{CH}_{4}$ column density of $6 \times$ $10^{15} \mathrm{~cm}^{-2}$ and a mean electron energy of $12 \pm 3 \mathrm{keV}$. This is a moderate energy in comparison with the $40-200 \mathrm{keV}$ determined from the analysis of Jupiter's STIS spectra of the main oval and high-latitude regions [Gérard et al., 2003; Gustin et al., 2004]. The bulk of the electron energy is deposited near the $2 \times 10^{-7}$ bar level, that is approximately $160 \mathrm{~km}$ below the altitude of the homopause in the model of Moses et al. [2000].

\section{Discussion}

[21] To summarize our findings based on a set of 15 images of the Saturn's south polar region:

[22] 1. Images show an inhomogeneous main oval around the south pole at $70^{\circ}-80^{\circ}$. Its morphology is quite variable and reminiscent of the Jovian FUV aurora. Auroral precipitation is generally stronger and more structured in the morning sector and can take the aspect of a spiral stucture. However, the aurora is occasionally brighter in the noon and afternoon sector. The arc is thinner in the morning sector and is located between $70^{\circ}$ and $75^{\circ}$, with local emission rates reaching $\sim 75 \mathrm{kR}$.

[23] 2. A region of enhanced emission is sometimes observed in the noon sector at higher latitude than the main oval. In this case, the morning arc appears to be weak or absent. The two situations may correspond to different states of the magnetosphere, possibly north or south orientation of the IMF $B_{z}$ component.

[24] 3. Weak emission of a few $\mathrm{kR}$ is also present inside the oval at high latitudes as a diffuse region of emission. Spectra of this region, although quite noisy, show a clear auroral signature.

[25] 4. The main oval brightness is $\sim 2$ orders of magnitude less than the Jovian aurora. Its emitted power is comparable to the $4 \times 10^{10} \mathrm{~W}$ previously observed with the WFPC2 camera in the north aurora. The total precipitated hemispheric power is generally in the range 20$140 \mathrm{GW}$, with a possible dependence on solar activity.

[26] 5. The spectra show little variation of the amount of methane absorption, suggesting that in the noon sector where they were observed, the auroral electron energy remains close to $10 \mathrm{keV}$. The bulk of this energy is deposited $\sim 160 \mathrm{~km}$ below the homopause where it is partly radiated away by thermal infrared emission and, combined with Joule heating, possibly affects the high-latitude thermal structure.

[27] The Voyager UVS observations of the south polar regions reported by Sandel and Broadfoot [1981] did not provide images of the aurora. The oval position at $78^{\circ}-$ $81.5^{\circ}$ near the dusk terminator was determined from a slit scan near disk, with uncertainties possibly associated with this mode. Observations in this sector made in October 1997 (Figure 2) are in good agreement with this range of latitudes. The global morphology described in this study confirms the main features of the WFPC2 observations reported by Trauger et al. [1998], although the higher sensitivity and resolution of STIS provides a considerable more detailed picture of Saturn's aurora. Analysis of the WFPC2 observations located auroral emission between $74^{\circ}$ and $79^{\circ} \mathrm{N}$, with a peak between $74^{\circ}$ and $79^{\circ}$ in the $0700-$ 0900 MLT sector. These values are somewhat larger than 
our results, where the main oval lies between $70^{\circ}$ and $78^{\circ} \mathrm{S}$ in this sector. Whether or not the difference is significant cannot be unambiguously determined. However, it should be noted that the observed poles were different and the viewing geometry was more favorable for the STIS images than for the WFPC2 data. The WFPC2 images were collected in October 1994 and 1995 when the planetary tilt was about $8^{\circ}$ and $2^{\circ}$, respectively, causing large uncertainties on the determination of the aurora's latitude. Finally, recent (unpublished) STIS images obtained in January 2004 show that the size of the oval decreases during periods of larger solar wind dynamic pressure. It may also have been the case when WFPC2 and UVS observations were made.

[28] The few observations available so far limit theoretical expectations for Saturn's aurora. In the case of Jupiter, the main auroral oval is believed to result from the breakdown in corotation of outward drifting plasma, driving currents in and out of Jupiter's ionosphere. In the region of upward current (downward electrons), field-aligned potentials presumably accelerate electrons to auroral energies, producing the main oval emissions [Hill, 2001; Cowley and Bunce, 2001; Grodent et al., 2003b]. A model for this process at Jupiter has recently been applied to the case of Saturn [Cowley and Bunce, 2003]. They showed that the upward current associated with subcorotation is not sufficiently intense to produce significant auroral acceleration and emission and that the expected latitude of such currents is located equatorward of the observed main oval. Instead, Cowley et al. [2004b] suggest that the auroral oval at Saturn corresponds to a ring of upward current bounding the region of open and closed field lines. In this model an electric potential drop is associated with the large current density carried by upflowing electrons. They estimate that an aurora of brightness from a few kR to a few tens of kR can be produced by precipitating magnetospheric electrons accelerated to a few $\mathrm{keV}$ to a few tens of $\mathrm{keV}$ energy if the current flows in a sufficiently narrow region, of order or less than $\sim 1000 \mathrm{~km}\left(1^{\circ}\right.$ latitude) wide. They also predict that the aurora should be significantly brighter on the dawnside of the oval than at dusk and additionally somewhat displaced toward dawn by the down-tail outflow at dusk. The calculated precipitated hemispheric power is $28 \mathrm{GW}$ in the south, to be compared with the observed 10-140 GW. The predicted main oval is near $75^{\circ}$ in the south, that is, a few degrees poleward of the observed location of the thin morning arc. With this exception, these model predictions are in good qualitative and quantitative agreement with the observations described in this work. The location of the morning arc is well predicted by model of field-aligned currents calculated on the basis of the corotation lag observed with Voyager. However, the afternoon more diffuse and less structured emission and noon spot are not accounted for in the current stage of the model. These features possibly stem from local time asymmetries associated with the Dungey [1961] and Vasyliunas [1983] cycles described by Cowley et al. [2003], which will enhance the flow shear, current, and precipitation near the open-closed field line boundary at dawn compared with other sectors. The presence of these features and the two different morphological aspects described before raises the question of solar wind-magnetosphere interactions as an alternative precipitation mechanism controlling both the brightness and the structure of the aurora. In particular, the morning arc fixed in local time may possibly be associated with Kelvin-Helmholtz instabilities at the magnetopause driving MHD waves [Galopeau et al., 1995]. These instabilities are expected to be enhanced in the morning sector where the relative velocity between the solar wind plasma and the corotating magnetospheric plasma is maximum. Another mechanism based on wave-particle interactions in the Titan plasma torus [Barbosa, 1990] would predict a longitudinally uniform aurora, in contrast with the distribution usually observed. More generally, both theory and observations thus suggest that Saturn's aurora is to some respect more Earthlike, connected to the distant magnetosphere close to the solar wind boundary.

[29] Neither the limited sample of images collected on 11 October 1997 nor the two-image sequences collected later provide compelling evidence for total or partial corotation of Saturn's aurora with the planet. However, these data do not necessarily imply that plasma rotation does not take place in the magnetospheric region mapping to the main oval or poleward. Our observations suggest that the longitudinal distribution of the auroral precipitation is at least partly controlled by local time-dependent processes. Similarly, this sample is too limited to provide evidence for a longitudinal sector of enhanced precipitation, as suggested by the Voyager UVS data. Such a longitudinal control would require observations of at least one or more complete planetary rotation.

[30] The presence of a bright spot in the noon sector and its location poleward of the main oval is consistent with a daytime cusp emission similar to the signature of reconnection-induced precipitation observed with the IMAGE-FUV Lyman- $\alpha$ imager in the Earth's ionosphere. In the terrestrial case it has been recently established that the dayside proton auroral spot is caused by solar wind protons leaking through and accelerated at the magnetopause following reconnection between solar wind and magnetospheric field lines [Phan et al., 2003]. When the $B_{z}$ component is oriented northward, the antiparallel reconnection site is located at the highlatitude magnetopause, causing the spot to move poleward of the main oval. For southward reconnection the cusp spot is located closer or on the main oval. A possible scenario is that for quiet solar conditions (or $B_{z}$ orientation) the main oval is located near $70^{\circ}$ and forms a quasi-continuous arc along the $70^{\circ}$ parallel with no FUV cusp signature. Instead, when solar activity increases (or $B_{z}$ flips orientation) the single arc structure is perturbed, leaving room to more diffuse and latitudinally extended emission and an enhanced dayside cusp signature. A polar "spot" or "flare" aurora of highly variable intensity, remaining persistently near local noon, has been suggested to be associated with the jovian cusp [Pallier and Prangé, 2001]. Bunce et al. [2004] noted that without acceleration, the largest precipitating energy fluxes are associated with magnetosheath protons, but these produce intensities well below those observed in the Jovian case. Therefore since acceleration must be present, they investigated the possibility that the emission is associated with pulsed reconnection at the dayside magnetopause. Although the details depend upon the assumptions for solar wind conditions, they concluded that the reconnection pulses produce bright "spots" or "arcs" extending along the Jovian open-closed field line boundary. The observa- 
tions of Saturn's aurora described in this study suggest more analogy with the Earth's than the Jovian situation. In particular, the two extreme structures described before, where the noon spot may be present or not, are possibly a signature of antiparallel reconnections occurring at different locations depending on the strength and orientation of the solar wind magnetic field at the daytime magnetopause.

[31] Acknowledgments. This work is based on observations made with the NASA/ESA Hubble Space Telescope. STScI is operated by the AURA, Inc. for NASA. The data were obtained from the Space Telescope Science Institute's (STScI) archives (programs 6854, 8117: J.T. Trauger, P.I., and 8158: R. Prangé, P.I.). J.C.G. and D.G. acknowledge support from the Belgian Fund for Scientific Research (FNRS). The ESA PRODEX program provided financial support for this research to the University of Liège.

[32] Arthur Richmond thanks Melissa McGrath and another reviewer for their assistance in evaluating this paper.

\section{References}

Barbosa, D. D. (1990), Auroral precipitation flux of ions and electrons in Saturn's outer magnetosphere, Planet. Space Sci., 38, 1295.

Ben Jaffel, L., V. Leers, and B. R. Sandel (1995), Dark auroral oval on Saturn discovered in Hubble Space Telescope ultraviolet images, Science, 269, 951 .

Broadfoot, A. L., et al. (1981), Extreme ultraviolet observations from Voyager 1 encounter with Saturn, Science, 212, 206.

Bunce, E. J., S. W. H. Cowley, and T. K. Yeoman (2004), Jovian cusp processes: Implications for the polar aurora, J. Geophys. Res., 109, A09S13, doi:10.1029/2003JA010280.

Clarke, J. T., H. W. Moos, S. K. Atreya, and A. L. Lane (1981), IUE detection of bursts of H Ly a emission from Saturn, Nature, 290, 226.

Connerney, J. E. P., L. Davis Jr., and D. L. Chenette (1984), Magnetic field models, in Saturn, edited by T. Gehrels and M. S. Matthews, p. 378, Univ. of Ariz. Press, Tucson, Ariz.

Cowley, S. W. H., and E. J. Bunce (2001), Origin of the main auroral oval in Jupiter's coupled magnetosphere-ionosphere system, Planet. Space Sci. 49, 1067.

Cowley, S. W. H., and E. J. Bunce (2003), Corotation-driven magnetosphere-ionosphere coupling currents in Saturn's magnetosphere and their relation to the auroras, Ann. Geophys., 21, 1691.

Cowley, S. W. H., E. J. Bunce, T. S. Stallard, and S. Miller (2003), Jupiter's polar ionospheric flows: Theoretical interpretation, Geophys. Res. Lett., 30(5), 1220, doi:10.1029/2002GL016030.

Cowley, S. W. H., E. J. Bunce, and J. M. O'Rourke (2004a), A simple quantitative model of plasma flows and currents in Saturn's polar ionosphere, J. Geophys. Res., 109, A05212, doi:10.1029/ 2003JA010375.

Cowley, S. W. H., E. J. Bunce, and R. Prangé (2004b), Saturn's polar ionospheric flows and their relation to the main auroral oval, Ann. Geophys., 21,1

Davis, L., Jr., and E. J. Smith (1990), A model of Saturn's magnetic field based on all available data, J. Geophys. Res., 95, 15,257.

Dols, V., J.-C. Gérard, J. T. Clarke, J. Gustin, and D. Grodent (2000), Diagnostics of the Jovian aurora deduced from ultraviolet spectroscopy: Model and HST/GHRS observation, Icarus, 147, 251.

Dungey, J. W. (1961), Interplanetary field and the auroral zones, Phys. Rev. Lett., 6, 47.

Galopeau, P., P. Zarka, and D. Lequéau (1995), Source location of Saturn's kilometric radiation: The Kelvin-Helmholtz instability hypothesis, J. Geophys. Res., 100, 26,397.

Geballe, T. R., M.-F. Jagod, and T. Oka (1993), Detection of $\mathrm{H}_{3}^{+}$emission lines in Saturn, Astrophys. J., 408, L109.

Gérard, J.-C., and V. Singh (1982), A model of energetic electrons and EUV emission in the Jovian and Saturnian atmospheres and implications, J. Geophys. Res., 87, 4525 .

Gérard, J.-C., V. Dols, D. Grodent, J. H. Waite, G. R. Gladstone, and R. Prangé (1995), Simultaneous observations of the Saturnian aurora and polar haze with the HST/FOC, Geophys. Res. Lett., 22, 2685.
Gérard, J.-C., D. Grodent, V. Dols, and J. H. Waite Jr. (1998), The longitudinal variation of the color ratio of the Jovian ultraviolet aurora: A geometric effect?, Geophys. Res. Lett., 25, 1601.

Gérard, J.-C., J. Gustin, D. Grodent, P. Delamere, and J. T. Clarke (2002), The excitation of the FUV Io tail on Jupiter: Characterization of the electron precipitation, J. Geophys. Res., 107(A11), 1394, doi:10.1029/ 2002JA009410.

Gérard, J.-C., J. Gustin, D. Grodent, J. T. Clarke, and A. Grard (2003), Spectral observations of transient features in the FUV Jovian polar aurora, J. Geophys. Res., 108(A8), 1319, doi:10.1029/2003JA009901.

Grodent, D., J. H. Waite, and J.-C. Gérard (2001), A self-consistent model of the Jovian auroral thermal structure, J. Geophys. Res., 106, 12,933.

Grodent, D., J. T. Clarke, J. H. Waite Jr., S. W. H. Cowley, J.-C. Gérard, and J. Kim (2003a), Jupiter's polar auroral emission, J. Geophys. Res., 108(A10), 1366, doi:10.1029/2003JA010017.

Grodent, D., J. T. Clarke, J. Kim, J. H. Waite Jr., and S. W. H. Cowley (2003b), Jupiter's main auroral oval observed with HST-STIS, J. Geophys. Res., 108(A11), 1389, doi:10.1029/2003JA009921.

Gustin, J., D. Grodent, J.-C. Gérard, and J. T. Clarke (2002), Spatially resolved far ultraviolet spectroscopy of the Jovian aurora, Icarus, 156, 91.

Gustin, J., J.-C. Gérard, D. Grodent, S. W. H. Cowley, J. T. Clarke, and A. Grard (2004), Energy-flux relationship in the Jovian aurora deduced from HST-STIS spectral observations, J. Geophys. Res., 109, doi:10.1029/2003JA010365, in press.

Hill, T. W. (2001), The Jovian auroral oval, J. Geophys. Res., 106, 8101.

Kaiser, M. L., M. D. Desch, W. S. Kurth, A. Lecacheux, F. Genova, B. M. Pederson, and D. R. Evans (1984), Saturn as a radio source, in Saturn, edited by T. Gehrels and M. S. Matthews, p. 378, Univ. of Ariz. Press, Tucson, Ariz.

McGrath, M. A., and J. T. Clarke (1992), H I Lyman alpha emission from Saturn (1980-1990), J. Geophys. Res., 103, 20,237.

Moses, J. I., B. Bézard, E. Lellouch, H. Feuchtgruber, G. R. Gladstone, and M. Allen (2000), Photochemistry of Saturn's atmosphere I. Hydrocarbon chemistry and comparisons with ISO observations, Icarus, 143, 244.

Pallier, L., and R. Prangé (2001), More on the structure of the high-latitude Jovian aurorae, Planet. Space Sci., 49, 1159.

Phan, T. D., et al. (2003), Extended magnetic reconnections at the Earth's magnetopause from detection of bi-directional jets, Nature, 404, 848 .

Sandel, B. R., and A. L. Broadfoot (1981), Morphology of Saturn's aurora, Nature, 292, 679 .

Sandel, B. R., et al. (1982), Extreme ultraviolet observations from the Voyager 2 encounter with Saturn, Science, 292, 679.

Shemansky, D. E., and J. M. Ajello (1983), The Saturn spectrum in the EUV: Electron excited hydrogen, J. Geophys. Res., 88, 459.

Smith, G. R., D. E. Shemansky, J. B. Holberg, A. L. Broadfoot, B. R. Sandel, and J. C. McConnell (1983), Saturn's upper atmosphere from the Voyager 2 EUV solar and stellar occultations, J. Geophys. Res., 88, 8667.

Stallard, T., S. Miller, G. E. Ballester, D. Rego, R. D. Joseph, and L. M. Trafton (1999), The $\mathrm{H}_{3}^{+}$latitudinal profile of Saturn, Astrophys. J., 521, L149.

Stallard, T., S. Miller, S. W. H. Cowley, and E. J. Bunce (2003), Jupiter's polar ionospheric flow: Measured intensity and veleocity variations poleward of the main auroral oval, Geophys. Res. Lett., 30(5), 1221, doi:10.1029/2002GL016031.

Trauger, J. T., et al. (1998), Saturn's hydrogen aurora: Wide field and planetary camera 2 imaging from the Hubble Space Telescope, J. Geophys. Res., 103, 20,237.

Vasyliunas, V. M. (1983), Plasma distribution and flow, in Physics of the Jovian Magnetosphere, edited by A. J. Dessler, p. 395, Cambridge Univ. Press, New York.

Waite, J. H., Jr., T. E. Cravens, J. U. Kozyra, A. F. Nagy, S. K. Atreya, and R. H. Chen (1983), Electron precipitation and related aeronomy of the Jovian thermosphere and ionosphere, J. Geophys. Res., 88, 6143.

J. T. Clarke, Center for Space Physics, Boston University, Boston, MA 02215, USA.(jclarke@bu.edu)

J.-C. Gérard, D. Grodent, J. Gustin, and A. Saglam, Laboratoire de Physique Atmosphérique et Planétaire, Université de Liège, B-4000 Liège, Belgium. (jc.gerard@ulg.ac.be; d.grodent@ulg.ac.be; j.gustin@ulg.ac.be) J. T. Trauger, Jet Propulsion Laboratory, Pasadena, CA 91109, USA. (jtt@lyot.jpl.nasa.gov) 